\title{
Manejo de la hipertensión arterial desde los servicios de atención primaria
}

\author{
Edgar Segarra E., Lorena E. Encalada T., Jorge L. García A.
}

Facultad de Ciencias Médicas, Universidad de Cuenca, Cuenca, Ecuador

Autor para correspondencia: esegarra@ucuenca.edu.ec

Fecha de recepción: 15 de enero de 2012 - Fecha de aceptación: 20 de febrero de 2012

\section{RESUMEN}

El objetivo de este estudio fue validar un modelo de Atención Primaria de Salud en pacientes hipertensos, en dos Centros de Salud de la ciudad de Cuenca, con el fin de: identificar los factores de riesgo, aplicar un programa de intervención educativa, evaluar la adherencia al tratamiento y los conocimientos, actitudes y prácticas (CAPs) relacionadas con la hipertensión arterial. Se realizó un estudio analítico prospectivo con un diseño cuasi-experimental. La muestra fue de tipo clúster e incluyó 100 pacientes en el grupo de intervención y 219 en el grupo de control. La intervención se aplicó durante 12 meses y consistió en actividades educativas, consultorios clínicos grupales y guías clínicas. La media de edad fue de 66,73 años para el grupo de intervención y de 69,26 años para el grupo control, con predominio del sexo femenino en ambos grupos $(75 \%$ y $71,7 \%)$ respectivamente. De los factores de riesgo estudiados presentaron significancia estadística la edad, la instrucción escolar y la adherencia al tratamiento. Se consiguió disminuir la presión arterial sistólica y diastólica en 13,26 y 2,19 $\mathrm{mmHg}$ respectivamente. La prevalencia de los factores de riesgo (tabaco, alcohol, sedentarismo) presentó una disminución estadísticamente significativa. Se incrementaron la adherencia $(\mathrm{p}=0,001)$ y el grado de CAPs $(\mathrm{p}=0,059)$. Se concluye que la intervención resultó efectiva para disminuir las cifras de tensión arterial sistólica y diastólica, para reducir algunos factores de riesgo, aumentar la adherencia al tratamiento y el grado de CAPs.

Palabras clave: Hipertensión arterial, servicios de Atención Primaria de Salud, factores de riesgo, Test de Batalla, Test de Morisky-Green.

\begin{abstract}
The aim of this study was to validate a Primary Health Care model for hypertensive patients in two health centers of the city of Cuenca; in order to identify risk factors, implement an educational intervention program, assess adherence to treatment and the Knowledge, Attitude and Practice (KAP) related to hypertension. We performed a prospective, analytical study with a quasi-experimental design. The sampling method was cluster sampling; included 100 patients in the intervention group and 219 in the control group. The intervention was implemented over 12 months and involved educational activities, group clinical consultation and clinical guidelines. The mean age was 66,73 years for the intervention group and 69,26 years for the control group, with female predominance in both groups $(75 \%$ and $71,7 \%)$ respectively. Studied risk factors that showed statistical significance were age, schooling and adherence to treatment. We achieved lower systolic and diastolic blood pressure at 13,26 and $2,19 \mathrm{mmHg}$ respectively. The prevalence of risk factors (snuff, alcohol, sedentary lifestyle) showed a statistically significant decrease. Also, adherence $(p=0,001)$ and the degree of KAP $(p=0,059)$ were increased. We conclude that the intervention was effective in reducing the levels of systolic and diastolic blood pressure, decreasing risk factors, increasing adherence to treatment and the degree of KAP.
\end{abstract}

Keywords: Hypertension, Primary Health Care Services, Risk Factors, Batalla test, Morisky-Green test. 


\section{INTRODUCCIÓN}

La hipertensión arterial es un trastorno muy frecuente (alrededor de mil millones de personas en todo el mundo) y como tal, es un factor de riesgo de aterosclerosis bien establecido, que predispone a enfermedad coronaria, accidente cerebrovascular, insuficiencia cardiaca, fibrilación auricular, diabetes mellitus, muerte súbita cardiaca, síndrome de insuficiencia aórtica y enfermedad renal crónica; además, puede causar la muerte y la discapacidad en pacientes de todas las edades (Colegio Americano de Cardiología, 2011).

En el Ecuador, al igual que en otros países de América Latina, las enfermedades crónicas no transmisibles han superado a las enfermedades infecciosas; según estadísticas nacionales la diabetes mellitus tipo II, las enfermedades cerebrovasculares y la hipertensión arterial (HTA) se encuentran entre las cinco primeras causas de muerte (INEC, 2011). La prevalencia de hipertensión arterial aumenta cuando está asociada a factores de riesgo tales como la obesidad, consumo de alcohol y sedentarismo, aunque no se ha demostrado fehacientemente su relación con el tabaco. En nuestro país la prevalencia de la HTA es de $28,7 \%$, lo que significa que aproximadamente tres de cada diez ecuatorianos son hipertensos; de estos, el $41 \%$ saben que son hipertensos, reciben tratamiento un $23 \%$ y tienen un control adecuado apenas el 6,7\% (Consenso Latinoamericano sobre HTA, 2001).

La hipertensión arterial es una enfermedad multifactorial compleja con factores genéticos, ambientales y demográficos participando en su prevalencia. Entre los factores demográficos se encuentra el envejecimiento de la población. La hipertensión en pacientes de edad avanzada ha llegado a constituir un complejo transtorno cardiovascular que afecta más a las mujeres que a los hombres y se produce en todos los países, razas y grupos étnicos (Rosas, 2008). Este grupo de pacientes es más propenso a tener hipertensión e hipertensión sistólica aislada, daño de órganos, eventos cardiovasculares, y son menos propensos a tener controlada su hipertensión (Colegio Americano de Cardiología, 2011; Bertomeu, 2003).

Entre las causas de elevada prevalencia de hipertensión arterial en Latinoamérica se señalan un bajo nivel socioeconómico y educacional, los procesos de aculturación y factores étnicos. El bajo nivel educacional contribuye a que no se reconozca esta enfermedad y no se la trate adecuadamente. Se ha demostrado una correlación inversa entre años de escolaridad con mortalidad cardiovascular y cerebral, correlación más evidente en las mujeres (Limansky, 2005).

La diferencia de prevalencia de la HTA en determinados grupos étnicos parece tener relación con factores socioculturales relacionados con las formas, condiciones y estilos de vida (Fasce, 2000). Las formas de vida se refieren a ciertas circunstancias de la sociedad en general que delimitan sus potencialidades económicas, políticas, culturales y por supuesto, su situación de salud. Por condiciones de vida se entienden las características de los grupos sociales (lo particular) agrupados en barrios urbanos o suburbanos; según clases: etnias, géneros, etc. Los estilos de vida (lo singular) hacen relación a las particularidades propias de las familias y los individuos (Breilh, 2010). El mayor soporte a la teoría de que las condiciones del medio ambiente podían constituir un importante factor en el desarrollo de la hipertensión arterial, lo constituyó la observación de que en las comunidades que conservaban un estilo de vida tribal y sin contactos con la cultura occidental la presión arterial era muy baja, no se elevaba con la edad y la hipertensión arterial era una enfermedad desconocida; sin embargo, la presión arterial aumentaba en cuanto esos sujetos entraban en contacto con la cultura occidental (Rodríguez Chamorro, 2008).

Las transiciones epidemiológicas y demográficas se acompañan de "transición nutricional" con modificaciones importantes en la dieta y la actividad física, además de incorporar ciertos hábitos como el tabaco y el alcohol. La transición nutricional es un cambio desde: una dieta pobre e intensa actividad física, hacia una situación con dieta hiperenergética, con mayor proporción de grasas, y en la cual el estilo sedentario es la regla (Martorelly y Stein, 2001).

Los factores que se han asociado con un control inadecuado de la hipertensión arterial se pueden resumir de la siguiente forma: 1. Relacionados con el paciente: falta de compromiso y adherencia al tratamiento por factores educativos, socioeconómicos, edad, deterioro cognitivo y evolución silenciosa de la enfermedad, que lleva a la suspensión de la medicación; 2. Relacionados con el 
tratamiento: manejo incorrecto de los medicamentos antihipertensivos, monoterapia, número diario de tomas del medicamento; 3. Factores relacionados con el equipo y la técnica utilizada para medir la tensión arterial; y 4. Factores relacionados con la gestión clínica: falta de cumplimiento de las guías internacionales para el manejo y tratamiento de los pacientes hipertensos (Sánchez, 2005).

La Constitución Política de la República del Ecuador en los artículos 350, 358 y 360 dispone que "el sistema garantizará la promoción de la salud, prevención y atención integral, familiar y comunitaria, con base en la Atención Primaria de Salud (APS)". El Centro de Salud es la estructura física y funcional donde se desarrolla una parte importante de las actividades de la APS de forma coordinada, integral, continuada, permanente y con base en el trabajo en equipo de los profesionales que actúan en el mismo. Dentro de sus actividades están la prevención de la enfermedad y promoción de la salud, así como los asistenciales curativos. Sin embargo, el médico de un Centro de Salud dependiente del estado se encuentra abrumado por atender la gran demanda de pacientes con morbilidad diversa, y no tiene el tiempo suficiente para una atención adecuada del paciente hipertenso.

La hipertensión arterial no debe ser considerado únicamente como un problema aislado de cifras de tensión arterial (TA) por encima de los valores normales; no sólo se deben tomar en cuenta las cifras tensionales, sino también la presencia de factores de riesgo concomitantes, así como aspectos personales, médicos y sociales del paciente, es decir, su perfil de riesgo cardiovascular global. Deben realizarse intervenciones destinadas a modificar aquellos factores de riesgo que sean susceptibles de ser modificados. Existen numerosos estudios sobre los beneficios de los programas educativos sobre el manejo de la hipertensión arterial. En un meta-análisis de 16 ensayos clínicos controlados se demostró que las intervenciones que utilizan asesoramiento y educación dirigidas al cambio conductual, pueden ser eficaces para reducir la mortalidad en las poblaciones hipertensas (Ebrahim y col., 2011).

Con estos antecedentes, se plantea la hipótesis: que un programa de intervención educativa en los pacientes hipertensos que se sometan a un tratamiento farmacológico adecuado y además modifiquen sus factores de riesgo, tendrían cifras tensionales más bajas y menos efectos colaterales. Con tales propósitos, se ejecutó un proyecto que tuvo como objetivo general desarrollar un modelo de APS en pacientes hipertensos, en dos Centros de Salud de la ciudad de Cuenca. Los objetivos específicos de este estudio fueron: identificar los factores de riesgo asociados a la hipertensión arterial, aplicar un programa de intervención educativa en los pacientes hipertensos, evaluar la adherencia al tratamiento y los conocimientos, actitudes y prácticas (CAPs) antes y después de la intervención.

\section{MÉTODOS}

\subsection{Población de estudio}

Este estudio analítico prospectivo con un diseño cuasi-experimental se realizó en un grupo de pacientes hipertensos registrados en dos Centros de Salud urbanos del Área $\mathrm{N}^{\circ} 2$ Miraflores de la ciudad de Cuenca, con características y metodología de trabajo similares. Ambos tenían un registro de pacientes hipertensos y contaban con sus respectivos clubes de hipertensos. Los dos Centros de Salud disponían de una planta estable de médicos y enfermeras, internos de medicina, estudiantes de enfermería y de nutrición. La muestra fue de tipo clúster. Del total de pacientes hipertensos registrados se seleccionaron 100 pacientes del primer Centro que conformaron el grupo de intervención y 219 del segundo Centro que constituyeron el grupo control. Los demás se descartaron porque no se conocía su dirección, los datos estaban incompletos, habían fallecido o estaban sujetos a criterios de exclusión. Se excluyeron del estudio los pacientes con diabetes mellitus, hipertensión arterial secundaria, aquellos que presentaban alguna enfermedad psíquica o incapacitante que les impedía el consentimiento informado. La fase de evaluación basal o preintervención se realizó durante el mes de febrero del 2009, mediante visitas domiciliarias previa cita telefónica. Durante la visita se procedió a registrar las variables del estudio, en las que se incluían la medición de los datos antropométricos y la toma de la presión arterial según normas internacionales. Durante la fase de 
intervención hubo una pérdida de 10 casos en el grupo de intervención y 29 casos en el grupo de control.

\subsection{Programa de intervención}

La fase de intervención se realizó desde 14 de mayo del 2010 hasta el 13 mayo del 2011, durante la cual se ejecutaron tres tipos de actividades: educativas, consultorios clínicos grupales y guías clínicas.

Actividades educativas

- Charlas informativas mensuales a los pacientes hipertensos, presentación de videos.

- Sesiones educativas con el personal del Centro de Salud (médicos, enfermeras, auxiliares de enfermería, internos de medicina y de enfermería).

- Retroalimentación: Presentación y análisis de los resultados al equipo de salud de la evaluación inicial de los dos Centros de Salud.

- Entrega de trípticos y folletos informativos a los pacientes hipertensos.

Consultorios clínicos grupales

Se implementó un modelo de atención intensiva en el que a las actividades educativas se adicionó el tratamiento médico personalizado de los pacientes hipertensos. Se conformaban grupos de 10 a 12 pacientes previa cita telefónica. En cada sesión se realizaron interacciones grupales, coordinadas por el educador, previas a la consulta médica. El médico del proyecto de investigación y el farmacéutico del Centro de Salud ajustaban las dosis de la medicación según las cifras de presión arterial de cada paciente. Las sesiones se realizaban los días viernes de cada semana, de 10 am a 13 pm. Además, se valoró la evolución de la presión arterial en el grupo de intervención mediante la medición sistemática de la cifras de presión arterial en cada una de las sesiones grupales.

Guías clínicas

Se elaboraron resúmenes informativos sobre el manejo de la hipertensión arterial en APS para el personal de salud.

Finalmente, se realizó una nueva evaluación postintervención en los dos Centros de Salud desde el 15 de mayo hasta el 13 de junio del 2011, analizando los mismos parámetros recogidos en la evaluación inicial y en el mismo grupo de pacientes en los que se había realizado la evaluación inicial.

\subsection{Definiciones y mediciones}

La variable dependiente fue la hipertensión arterial. Como variables independientes se consideraron la actividad física, el índice de masa corporal (IMC), el consumo de sal, la ingesta de alcohol, el tabaquismo, la adherencia al tratamiento y los conocimientos actitudes y prácticas sobre hipertensión arterial (CAPs).

Los estadios de la HTA se clasificaron de acuerdo al JNV-7 en cuatro tipos: normal $(<120 / 80$ mmHg); prehipertensos (120-139/80-89); HTA estadio I (140-159/90-99); HTA estadio II (> 160/100) (Joint National Committee on Prevention, Detection, Evaluation and Treatment of High Blood Pressure, 2004). La medición de la presión arterial se realizó con esfigmomanómetros aneroides (Welch Allyn ${ }^{\circledR}$ ) debidamente calibrados, luego de cinco minutos de reposo, en posición sentado, con el antebrazo derecho apoyado sobre una mesa, la espalda apoyada en un respaldo y ambos pies colocados sobre el suelo. Se calculó el promedio de dos mediciones de la TA obtenidas con un intervalo de 5 minutos, utilizando como referencia el primer sonido de Korotkoff para la presión sistólica y la quinta fase para la diastólica.

Con respecto a la actividad física se consideró como persona activa aquella que practicaba alguna actividad aeróbica (caminar, trotar, gimnasia, ciclismo, baile) durante 30 minutos (no necesariamente consecutivos), durante 5 días a la semana.

El índice de masa corporal (IMC) llamado también Índice de Quetelec se calculó de acuerdo a la fórmula peso $(\mathrm{kg})$ por talla $\left(\mathrm{m}^{2}\right)$. Se consideró como normal el rango 18,5-24,9 $\mathrm{kg} \mathrm{m}^{-2}$; sobrepeso $25-$ $29,9 \mathrm{~kg} \mathrm{~m}^{-2}$; obesidad clase I: $30-34 \mathrm{~kg} \mathrm{~m}^{-2}$; obesidad clase II: $35-39,9 \mathrm{~kg} \mathrm{~m}^{-2}$; obesidad clase III: $\geq 40$ $\mathrm{kg} \mathrm{m}^{-2}$. El peso se determinó a partir del medio kilo más cercano, en una báscula clínica (SECA), con 
el paciente descalzo y con la mínima cantidad de ropa posible respetando su intimidad. La estatura se midió utilizando una cinta métrica metálica graduada en centímetros de 2,50 de largo, apoyada sobre la pared, haciendo coincidir el cero con el plano horizontal (piso). El registro de la altura se realizó con el paciente sin calzado, en extensión máxima midiendo la distancia entre el piso y el vértex craneal. Para ello la posición de la cabeza se colocó en el plano de Frankfort (Mazza, 2003). El evaluador se colocó delante del paciente y se solicitó que coloque los pies y las rodillas juntas, talones, cara posterior de glúteos y cabeza fijados a la cinta métrica; se le pidió que inspire profundamente y luego se le solicitó relajación y estiramiento. En ese momento se colocó un objeto triangular sobre el vértex y haciendo contactar con la cinta se midió la talla en centímetros.

La ingesta excesiva de sal se determinó interrogando al paciente si acostumbra agregar sal a los alimentos, o si consumía sal en una cantidad aproximada de 6 gramos (una cucharadita de té). Se consideró que existía una ingesta excesiva de alcohol cuando el paciente hipertenso aseguraba que ingería más de 2 tragos diarios ( $\geq 60 \mathrm{ml} \mathrm{día}{ }^{-1}$ de etanol en el hombre y $\geq 30 \mathrm{ml} \mathrm{día}^{-1}$ en la mujer). La variable tabaquismo se codificó de manera dicotómica en fumador y no fumador; se consideró fumador a toda persona que en ese momento fumaba, aunque fuera ocasionalmente.

Se consideró que existía adherencia al tratamiento cuando contestaban en forma correcta todas las preguntas del test de Morisky- Green y no-adherencia a quienes contestaron en forma incorrecta una o más preguntas. El paciente tenía suficientes conocimientos, actitudes y prácticas (CAPs) sobre su enfermedad cuando respondía en forma correcta todas las preguntas del Test de Batalla y como carente de conocimientos sobre su enfermedad cuando no respondía correctamente a partir de 1 a 3 preguntas del test. Con respecto al nivel educativo se agrupó a los participantes en analfabetos, educación primaria, secundaria y superior. Las categorías de educación representan hitos educativos reconocidos que influyen en los ingresos, el tipo de educación y la posición socioeconómica en la sociedad (Loucks, 2011).

\subsection{Análisis estadístico}

Los datos recolectados fueron ingresados en una base diseñada para tal efecto en el software Excel 2007. El procesamiento estadístico se realizó en el software estadístico SPSS v.15. Los datos fueron resumidos en tablas y gráficos de acuerdo con el tipo de variables. Previo al análisis definitivo se procedió a un análisis exploratorio de los datos con el fin de realizar los ajustes necesarios a los mismos. Para el análisis estadístico definitivo se utilizó medidas de frecuencia relativa para las variables cualitativas. En el caso de las variables cuantitativas se utilizó medidas de tendencia central (promedio aritmético y desvío estándar, error típico). Para determinar la homogeneidad de los grupos de intervención y control se utilizó la diferencia de medias. Para el análisis descriptivo de los datos se utilizó: el promedio, la mediana, desviación estándar y rango intercuartilar (de acuerdo a la normalidad de las variables determinadas por un valor $\mathrm{p}$ de Shapiro Wilk $\mathrm{p}>0,05)$. Para determinar asociación entre variables se utilizó la prueba $\mathrm{t}$ (variables contínuas de distribución normales) o U de Mann-Whitney (variables contínuas de distribución no normal) con un nivel de significancia de 0,05. $\chi^{2}$ de Pearson (variables nominales) o Test de Fisher, según corresponda; y, Tau "b" o "c" de Kendal para variables ordinales; el $\chi^{2}$ de McNemar para evaluar los cambios luego de la intervención, todas ellas con un nivel de confianza del $95 \%$.

\section{RESULTADOS Y DISCUSIÓN}

Las características generales de los 319 pacientes hipertensos estudiados (100 del grupo de intervención y 219 del grupo control) se pueden observar en la Tabla 1. La media de edad fue 66,73 años para el grupo de intervención y 69,26 para el grupo control. Este hallazgo se corresponde con lo planteado por la literatura, donde se describe que: a mayor edad, mayor es el riesgo de padecer hipertensión arterial y que por ende aumenta progresivamente con la edad (Saieh y Zehnder, 2008). Se ha comprobado que la presión arterial promedio tiende a aumentar de manera progresiva a medida que el individuo envejece; este incremento de la frecuencia de hipertensión arterial sobre la base de la 
edad, es siempre mayor para la sistólica que para la diastólica (López, 2002; Poveda, 2006). En la Tabla 2 se aprecia que la prevalencia de la HTA es mucho mayor $(74,61 \%)$ en el grupo $>59$ años. Se considera un factor de riesgo la edad mayor de 55 años en hombres y mayor de 65 años en mujeres (Marso y col., 2002). En este estudio se encontró una relación estadísticamente significativa (p > 0,05) entre la edad y la HTA (Tabla 2).

Tabla 1. Variables descriptivas basales de edad, sexo, factores de riesgo, cifras de TA en pacientes hipertensos de dos Centros del Área de Salud No 2 Miraflores, Cuenca, 2010.

\begin{tabular}{|c|c|c|c|c|c|}
\hline \multirow{2}{*}{ Variable } & \multirow{2}{*}{\multicolumn{2}{|c|}{$\begin{array}{c}\text { Intervención } \\
\mathrm{n}=100\end{array}$}} & \multirow{2}{*}{\multicolumn{2}{|c|}{$\begin{array}{c}\text { Control } \\
n=219\end{array}$}} & \multirow{2}{*}{$\mathrm{p}$} \\
\hline & & & & & \\
\hline Edad, años, media \pm DE & 66,73 & $(11,56)$ & 69,26 & $(13,54)$ & NS \\
\hline Edad varones, años $\pm \mathrm{DE}$ & 69,84 & $(11,29)$ & 70,40 & $(14,71)$ & NS \\
\hline Edad mujeres, años $\pm \mathrm{DE}$ & 65,69 & $(11,53)$ & 68,80 & $(13,06)$ & NS \\
\hline Varones, n \% & 25 & $(25,00)$ & 62 & $(28,31)$ & NS \\
\hline $\mathrm{PAS}$, media $\pm \mathrm{DE}$ & 137,29 & $(18,67)$ & 134,76 & $(17,81)$ & NS \\
\hline $\mathrm{PAD}$, media $\pm \mathrm{DE}$ & 80,48 & $(10,46)$ & 78,31 & $(11,22)$ & NS \\
\hline $\mathrm{TA}<140 /<90, \mathrm{n} \%$ & 60 & $(40,00)$ & 142 & $(64,84)$ & NS \\
\hline Obesidad (IMC > 30), n \% & 44 & $(44,00)$ & 61 & $(27,85)$ & NS \\
\hline Tabaco, n \% & 1 & $(1,00)$ & 19 & $(8,68)$ & $<0,05$ \\
\hline Consumo de sal $>6 \mathrm{~g} \mathrm{~d}^{-1}, \mathrm{n} \%$ & 36 & $(36,00)$ & 64 & $(29,22)$ & NS \\
\hline Ingesta de alcohol $>60 \mathrm{ml} \mathrm{d}^{-1}, \mathrm{n} \%$ & 8 & $(8,00)$ & 28 & $(12,79)$ & NS \\
\hline Sedentarismo, n \% & 52 & $(52,00)$ & 103 & $(47,03)$ & NS \\
\hline Adherencia al tratamiento, $\mathrm{n} \%$ & 45 & $(45,00)$ & 112 & $(51,10)$ & NS \\
\hline CAPs, (Si) n \% & 51 & $(51,00)$ & 110 & $(50,23)$ & NS \\
\hline
\end{tabular}

En relación con el género, el porcentaje de varones fue $25 \%$ en el grupo de intervención y $28,31 \%$ en el grupo control (Tabla 1), lo que significa que la mayor parte de pacientes hipertensos fueron de sexo femenino con una relación aproximada de 3:1, datos que coinciden con numerosos trabajos de investigación en nuestro medio y en APS (Poveda, 2006; Gómez-Marcos y col., 2006; Valdivieso, 2008; Coca Payeros, 2005). La prevalencia de hipertensión arterial es menor en mujeres que en hombres hasta los 45 años de edad, es similar en ambos sexos de 45 a 64 años de edad y es mucho mayor en mujeres por encima de los 65 años (Lamar y Hill, 2002). Los cambios hormonales después de la menopausia con elevación de la presión arterial son controvertidos. Por el contrario, hay una fuerte evidencia de estudios prospectivos longitudinales en donde se demuestra que la elevación de la presión arterial relacionada con la menopausia depende de un mayor índice de masa corporal y el envejecimiento, en lugar de una insuficiencia ovárica por si misma (Pimenta y col., 2006).

Varios estudios epidemiológicos reportan una mayor incidencia de HTA en pacientes con pocos años de escolaridad (Loucks, 2011). En el estudio de Framingham (Feinleib y col., 1983) se demostró que el nivel de educación es uno de los marcadores del estado socioeconómico y está relacionado en forma inversa con la prevalencia e incidencia de hipertensión arterial y con las complicaciones y evolución de ésta. Esta relación es más evidente en mujeres que en hombres. Al valorar el grado de instrucción e HTA se comprobó una asociación estadísticamente significativa (Tabla 2); hubo un $5,96 \%$ de pacientes hipertensos analfabetos, además el 83,7\% de la población hipertensa tuvo menos de 12 años de educación formal y muy pocos habían terminado sus estudios universitarios. Este bajo nivel de instrucción podría explicar la falta de adherencia al tratamiento y el desconocimiento por parte de la población sobre su enfermedad, que se detectó al inicio del proyecto mediante la aplicación del test de Morisky-Green, con un valor de p > 0,05 (Tabla 2). La educación se asocia con la mejoría en el control de la presión arterial; un nivel de educación menor al bachillerato es un factor de riesgo independiente y un reto para la "alfabetización de la salud" (Loucks, 2011).

En la Tabla 3 se evidencian los estadios de HTA antes de la intervención; la prevalencia de pacientes con TA normal fue apenas el 13,48\%, en tanto que el estadio 2 fue 36,68\%. Cuanto mayor sea la cifra de TA en los adultos mayores, se incrementa el riesgo de enfermedad cerebro y 
cardiovascular. Sin embargo, se asocia también a demencia lo que agrega una carga ulterior de discapacidad a las poblaciones envejecidas (Poveda, 2006). El impacto de la estrategia denominada

Tabla 2. Factores de riesgo y Diagnóstico de TA en pacientes hipertensos de dos Centros del Área de Salud No 2 Miraflores, Cuenca, 2010.

\begin{tabular}{|c|c|c|c|c|c|c|c|c|}
\hline \multirow[b]{2}{*}{ Variable } & & \multicolumn{4}{|c|}{ Diagnóstico de HTA } & \multirow[b]{2}{*}{$\begin{array}{c}\text { Total } \\
\mathrm{n}=319\end{array}$} & \multirow[b]{2}{*}{$(\%)$} & \multirow[b]{2}{*}{$\mathrm{p}$} \\
\hline & & $\begin{array}{l}\text { Normal } \\
\mathrm{n}=43\end{array}$ & $(\%)$ & $\begin{array}{c}\text { HTA } \\
n=276\end{array}$ & $(\%)$ & & & \\
\hline \multirow{3}{*}{ Edad } & $18-39$ & 0 & 0,00 & 1 & 0,36 & 1 & 0,31 & $<0,05$ \\
\hline & $40-59$ & 11 & 25,58 & 69 & 25,00 & 80 & 25,08 & \\
\hline & $>59$ & 32 & 74,42 & 206 & 74,64 & 238 & 74,61 & \\
\hline \multirow{2}{*}{ Sexo } & Femenino & 30 & 69,77 & 202 & 73,19 & 232 & 72,73 & NS \\
\hline & Masculino & 13 & 30,23 & 74 & 26,81 & 87 & 27,27 & \\
\hline \multirow{4}{*}{ Instrucción } & Analfabeto & 2 & 4,65 & 17 & 6,16 & 19 & 5,96 & $<0,05$ \\
\hline & Primaria & 22 & 51,16 & 148 & 53,62 & 170 & 53,29 & \\
\hline & Secundaria & 14 & 32,56 & 83 & 30,07 & 97 & 30,41 & \\
\hline & Superior & 5 & 11,63 & 28 & 10,14 & 33 & 10,34 & \\
\hline \multirow{2}{*}{$\begin{array}{l}\text { Ejercicio } \\
\text { físico }\end{array}$} & No & 17 & 39,53 & 138 & 50,00 & 155 & 48,59 & NS \\
\hline & $\mathrm{Si}$ & 26 & 60,47 & 138 & 50,00 & 164 & 51,41 & \\
\hline \multirow{2}{*}{$\begin{array}{l}\text { Consume } \\
6 \mathrm{~g} \text { de sal }\end{array}$} & No & 31 & 72,09 & 188 & 68,12 & 219 & 68,65 & NS \\
\hline & $\mathrm{Si}$ & 12 & 27,91 & 88 & 31,88 & 100 & 31,35 & \\
\hline \multirow{2}{*}{$\begin{array}{l}\text { Ingesta de } \\
\text { alcohol }\end{array}$} & No & 38 & 88,37 & 245 & 88,77 & 283 & 88,71 & NS \\
\hline & $\mathrm{Si}$ & 5 & 11,63 & 31 & 11,23 & 36 & 11,29 & \\
\hline \multirow{2}{*}{ Fuma } & No & 42 & 97,67 & 257 & 93,12 & 299 & 93,73 & $\mathrm{NS}$ \\
\hline & $\mathrm{Si}$ & 1 & 2,33 & 19 & 6,88 & 20 & 6,27 & \\
\hline \multirow{2}{*}{$\begin{array}{l}\text { Estado } \\
\text { nutricional }\end{array}$} & Normal & 35 & 81,40 & 193 & 69,93 & 228 & 71,47 & $\mathrm{NS}$ \\
\hline & Obesidad & 8 & 18,60 & 83 & 30,07 & 91 & 28,53 & \\
\hline \multirow{2}{*}{$\begin{array}{l}\text { Morisky- } \\
\text { Green }\end{array}$} & Adherencia & 30 & 69,80 & 12 & 46,00 & 162 & 50,80 & $<0,05$ \\
\hline & No adherer & 13 & 30,20 & 149 & 54,00 & 157 & 49,20 & \\
\hline Test de & No & 19 & 44,19 & 139 & 50,36 & 158 & 49,53 & NS \\
\hline Batalla & $\mathrm{Si}$ & 24 & 55,81 & 137 & 49,64 & 161 & 50,47 & \\
\hline
\end{tabular}

"Consultorios Clínicos Grupales" se refleja en la disminución de las cifras tensionales (Tabla 4), tanto de la presión arterial sistólica como la diastólica $(13,26$ y $2,19 \mathrm{mmHg}$ respectivamente) en el grupo intervenido, mientras que dichas variables se habían incrementado en el grupo control 2,96/3,26 mmHg. En un estudio similar realizado en España el efecto atribuido a la intervención fue un descenso de $9,72 \mathrm{mmHg}$ en la presión arterial sistólica y 3,84 $\mathrm{mmHg}$ en la diastólica; en el control ascendieron 1,56/0,13 respectivamente (Gómez-Marcos y col., 2006). Este resultado también se puede apreciar en el número de pacientes con presión arterial normal que se incrementó de 8 a 51 en el grupo intervenido y en la disminución de hipertensos en estadio II.

En el estudio que se analiza, la prevalencia de pacientes hipertensos con sobrepeso fue $44 \%$ y obesidad 30\% en el grupo intervenido (Tabla 3), con valores muy parecidos en el grupo control. En estudios similares la prevalencia de obesidad en pacientes hipertensos varía entre $35 \%$ y $40 \%$. (Rodríguez, 2009). El sobrepeso y la obesidad han sido identificados como los determinantes más importantes de hipertensión. Los cambios metabólicos y hormonales en la obesidad e hipertensión arterial se asocian a disfunción endotelial y aterosclerosis prematura. En el estudio Framingham (Feinleib y col., 1983) se encontró que un $10 \%$ de aumento del peso corporal produce un incremento de $7 \mathrm{mmHg}$ en la presión sistólica. La prevalencia de hipertensión en el NHANES II fue 2,9 veces mayor en los obesos que en los no obesos adultos (Díaz, 2002). 
A diferencia de otros estudios en donde la prevalencia de tabaquismo en la población hipertensa alcanza cifras de 35\% o más (Baena y col., 2005), en el presente estudio se encontró un porcentaje de $6,27 \%$ de pacientes que fumaban antes de la intervención (Tabla 3), pero luego estos valores disminuyeron en ambos grupos, lo que se reflejó en un nivel de significancia 0,025 (Tabla 4).

Tabla 3. Factores de riesgo basales, en pacientes hipertensos de dos Centros del Área de Salud № 2 Miraflores, Cuenca, 2010.

\begin{tabular}{|c|c|c|c|c|c|c|c|}
\hline \multirow[t]{2}{*}{ Variable } & & \multicolumn{2}{|c|}{$\begin{array}{c}\text { Intervención } \\
\mathrm{n}=100\end{array}$} & \multicolumn{2}{|c|}{$\begin{array}{l}\text { Control } \\
\mathrm{n}=219\end{array}$} & \multirow[t]{2}{*}{ Total } & \multirow[t]{2}{*}{$(\%)$} \\
\hline & & $\mathrm{n}$ & $(\%)$ & $\mathrm{n}$ & $(\%)$ & & \\
\hline Consume & No & 64 & 64,00 & 155 & 70,78 & 219 & 68,65 \\
\hline $6 \mathrm{~g}$ de sal & $\mathrm{Si}$ & 36 & 36,00 & 64 & 29,22 & 100 & 31,35 \\
\hline \multirow{2}{*}{ Ejercicio físico } & No & 52 & 52,00 & 103 & 47,03 & 155 & 48,59 \\
\hline & $\mathrm{Si}$ & 48 & 48,00 & 116 & 52,97 & 164 & 51,41 \\
\hline \multirow{2}{*}{ Fuma } & No & 99 & 99,00 & 200 & 91,32 & 299 & 93,73 \\
\hline & $\mathrm{Si}$ & 1 & 1,00 & 19 & 8,68 & 20 & 6,27 \\
\hline \multirow{4}{*}{ Estado nutricional } & Desnutrición & 1 & 1,00 & 1 & 0,46 & 2 & 0,63 \\
\hline & Normal & 25 & 25,00 & 58 & 26,48 & 83 & 26,02 \\
\hline & Sobrepeso & 44 & 44,00 & 99 & 45,21 & 143 & 44,83 \\
\hline & Obeso & 30 & 30,00 & 61 & 27,85 & 91 & 28,53 \\
\hline \multirow{2}{*}{ Test de Batalla } & No & 49 & 49,00 & 109 & 49,77 & 158 & 49,53 \\
\hline & $\mathrm{Si}$ & 51 & 51,00 & 110 & 50,23 & 161 & 50,47 \\
\hline \multirow[b]{2}{*}{ Morisky-Green } & Adherencia & 45 & 45,00 & 112 & 51,10 & 157 & 49.20 \\
\hline & No adherencia & 55 & 55,00 & 107 & 48,90 & 162 & 51,80 \\
\hline \multirow[b]{2}{*}{ Ingesta de alcohol } & No & 92 & 92,00 & 191 & 87,21 & 283 & 88,71 \\
\hline & $\mathrm{Si}$ & 8 & 8,00 & 28 & 12,79 & 36 & 11,29 \\
\hline \multirow{4}{*}{$\begin{array}{l}\text { Diagnóstico de } \\
\text { HTA }\end{array}$} & Normal & 8 & 8,00 & 35 & 15,98 & 43 & 13,48 \\
\hline & Prehipertensión & 51 & 51,00 & 96 & 43,84 & 147 & 46,08 \\
\hline & HTA: estadio 1 & 1 & 1,00 & 11 & 5,02 & 12 & 3,76 \\
\hline & HTA: estadio 2 & 40 & 40,00 & 77 & 35,16 & 117 & 36,68 \\
\hline \multirow{3}{*}{ Edad } & $18-39$ & 0 & 0,00 & 1 & 0,46 & 1 & 0,31 \\
\hline & $40-59$ & 27 & 27,00 & 53 & 24,20 & 80 & 25,08 \\
\hline & $>59$ & 73 & 73,00 & 165 & 75,34 & 238 & 74,61 \\
\hline \multirow{4}{*}{ Instrucción } & Analfabeto & 10 & 10,00 & 9 & 4,11 & 19 & 5,96 \\
\hline & Primaria & 65 & 65,00 & 105 & 47,95 & 170 & 53,29 \\
\hline & Secundaria & 20 & 20,00 & 77 & 35,16 & 97 & 30,41 \\
\hline & Superior & 5 & 5,00 & 28 & 12,79 & 33 & 10,34 \\
\hline
\end{tabular}

En la Tabla 5 se describen cada una de las variables estudiadas luego de la intervención, en donde se puede apreciar el incremento porcentual de prácticas favorables hacia la disminución de los factores de riesgo frente a la hipertensión si se compara con la Tabla 3. Las variables que mayor cambio presentaron fueron el consumo de sal, la actividad física y los CAPs. La adherencia al tratamiento subió del $45 \%$ al $80 \%$.

Si bien los estudios epidemiológicos y clínicos demuestran asociación entre ingesta de sal e hipertensión arterial, llama la atención que aún en las poblaciones que consumen las más altas ingestas de sal, existen porcentajes importantes de individuos que no desarrollan hipertensión, que pertenecen al grupo de resistentes a la sal (Jiang y col., 2002). Esto demuestra que la alta ingesta de sal no es la causa, sino el gatillo de la hipertensión actuando sobre las características individuales. La hipertensión geriátrica es habitualmente sal-sensible, con una alta frecuencia de hipertensión sistólica aislada (Valdéz, 2009). En el estudio que se analiza, en donde la mayor parte de la población es de la tercera edad, se encontró un porcentaje de $36 \%$ de pacientes que declararon consumir más de $6 \mathrm{~g}$ diarios de sal en el grupo intervenido. Después de la intervención, el porcentaje de consumidores excesivos de sal había disminuido al 26\%, mientras que en el grupo control los valores se 
incrementaron del $29,22 \%$ al $46,84 \%$; se encontró una p de 0,026 con respecto al consumo de sal antes y después de la intervención (Tabla 6).

Tabla 4. Variables post-intervención de factores de riesgo y cifras de TA en pacientes hipertensos de dos Centros del Área de Salud No 2 Miraflores, Cuenca, 2011.

\begin{tabular}{|c|c|c|c|c|c|}
\hline \multirow{2}{*}{$\begin{array}{l}\text { Variable } \\
\text { PAS, media } \pm \text { DE }\end{array}$} & \multicolumn{2}{|c|}{$\begin{array}{c}\text { Intervención } \\
\mathrm{n}=100\end{array}$} & \multicolumn{2}{|c|}{$\begin{array}{l}\text { Control } \\
\mathrm{n}=219\end{array}$} & \multirow{2}{*}{$\begin{array}{l}\mathrm{P} \\
0,000\end{array}$} \\
\hline & 124,03 & 12,39 & 137,71 & 18,02 & \\
\hline $\mathrm{PAD}$, media $\pm \mathrm{DE}$ & 78,29 & 9,96 & 81,57 & 81,57 & 0,023 \\
\hline $\mathrm{TA}<140 /<90, \mathrm{n} \%$ & 95 & 95,00 & 148 & 77,89 & 0,000 \\
\hline Obesidad (IMC > 30), n \% & 39 & 39,00 & 52 & 27,37 & 0,003 \\
\hline Tabaco, n \% & 0 & 0,00 & 7 & 3,68 & \\
\hline Consumo de sal $>6 \mathrm{~g} \mathrm{~d}^{-1}, \mathrm{n} \%$ & 26 & 26,00 & 89 & 46,84 & 0,001 \\
\hline Ingesta de alcohol $>60 \mathrm{ml} \mathrm{d}^{-1}, \mathrm{n} \%$ & 5 & 5,00 & 5 & 2,63 & 0,190 \\
\hline Sedentarismo, n \% & 33 & 33,00 & 84 & 44,21 & 0,064 \\
\hline Adherencia al tratamiento, $\mathrm{n} \%$ & 80 & 80,00 & 114 & 60,00 & 0,001 \\
\hline CAPs, $(\mathrm{Si}) \mathrm{n} \%$ & 84 & 84,00 & 69 & 36,32 & 0,000 \\
\hline
\end{tabular}

Tabla 5. Factores de riesgo post-intervención, en pacientes hipertensos de dos Centros del Área de Salud No 2 Miraflores, Cuenca, 2011.

\begin{tabular}{|c|c|c|c|c|c|c|c|}
\hline \multirow[t]{2}{*}{ Variable } & & \multicolumn{2}{|c|}{$\begin{array}{l}\text { Intervención } \\
\mathrm{n}=100\end{array}$} & \multicolumn{2}{|c|}{$\begin{array}{l}\text { Control } \\
\mathrm{n}=219 \\
\end{array}$} & \multirow[t]{2}{*}{ Total } & \multirow[t]{2}{*}{$(\%)$} \\
\hline & & $\mathrm{n}$ & $(\%)$ & $\mathrm{n}$ & $(\%)$ & & \\
\hline Consume & No & 74 & 74,0 & 101 & 53,16 & 175 & 60,34 \\
\hline $6 \mathrm{~g} \mathrm{de} \mathrm{sal}$ & $\mathrm{Si}$ & 26 & 26,0 & 89 & 46,84 & 115 & 39,66 \\
\hline \multirow[b]{2}{*}{ Ejercicio físico } & No & 33 & 33,0 & 84 & 44,21 & 117 & 40,34 \\
\hline & $\mathrm{Si}$ & 67 & 67,0 & 106 & 55,79 & 173 & 59,66 \\
\hline \multirow{2}{*}{ Fuma } & No & 96 & 96,0 & 183 & 96,32 & 279 & 96,21 \\
\hline & $\mathrm{Si}$ & 0 & 0,0 & 7 & 3,68 & 7 & 2,41 \\
\hline \multirow{4}{*}{ Estado nutricional } & Bajo peso & 1 & 1,0 & 6 & 3,16 & 7 & 2,41 \\
\hline & Normal & 15 & 15,0 & 52 & 27,37 & 67 & 23,10 \\
\hline & Sobrepeso & 45 & 45,0 & 80 & 42,11 & 125 & 43,10 \\
\hline & Obesidad & 39 & 39,0 & 52 & 27,37 & 91 & 31,38 \\
\hline \multirow{2}{*}{ Test Batalla } & No & 16 & 16,0 & 121 & 63,68 & 137 & 47,24 \\
\hline & $\mathrm{Si}$ & 84 & 84,0 & 69 & 36,32 & 153 & 52,76 \\
\hline \multirow{2}{*}{ Morisky-Green } & Adherentes & 80 & 80,0 & 114 & 60,00 & 194 & 66,90 \\
\hline & No adherentes & 20 & 20,0 & 76 & 40,00 & 96 & 31,10 \\
\hline \multirow{3}{*}{ Ingesta de alcohol } & No & 94 & 94,0 & 185 & 97,37 & 279 & 96,21 \\
\hline & $\mathrm{Si}$ & 5 & 5,0 & 5 & 2,63 & 10 & 3,45 \\
\hline & Controlada & 95 & 95,0 & 148 & 77,89 & 243 & 83,79 \\
\hline \multirow{4}{*}{ Diagnóstico de HTA } & Normal & 51 & 51,0 & 66 & 34,74 & 117 & 40,34 \\
\hline & Pre-hipertensión & 44 & 44,0 & 82 & 43,16 & 126 & 43,45 \\
\hline & HTA estado I & 5 & 5,0 & 35 & 18,42 & 40 & 13,79 \\
\hline & HTA estado 2 & 0 & 0,0 & 7 & 3,68 & 7 & 2,41 \\
\hline
\end{tabular}

Diversos estudios de cohortes con más de 5000 participantes han demostrado el aumento de incidencia de hipertensión arterial entre los sujetos sedentarios, independientemente de otros factores de riesgo (Varo y col., 2003; Pérez-García y col., 2002; Molina, 2008). En la Tabla 6 se demuestra el grupo de intervención con $52 \%$ de pacientes que se declararon sedentarios, aunque esta cifra disminuyó a $33 \%$ después de la mediación.

Según los resultados de diversas investigaciones, el test de Morisky-Green es una buena alternativa en APS para identificar pacientes hipertensos con riesgo de no cumplimiento del 
tratamiento. Al iniciarse el programa, el $45 \%$ de la población de estudio refirió adherencia al tratamiento, y al finalizar lo hizo el $80 \%$ (Tabla 6). En un trabajo similar realizado en España 51\% eran cumplidores (De Frutos y Lorenz, 2008) mientras que otro realizado en el Brasil refiere un 23\% de adhesión al tratamiento (Dosse y Bernardi, 2009). Otro estudio destinado a investigar este asunto, realizado en Argentina, refiere 62\% de adherencia antes de la intervención y $77 \%$ después de esta (Ferrera y col., 2010). Es posible que haya tenido un rol preponderante la intervención realizada en los pacientes agrupados en el "Club de Hipertensos", en donde se realizan hasta la fecha sesiones de gimnasia y charlas educativas sobre la importancia de la adherencia al tratamiento.

Para cuantificar los conocimientos, actitudes y prácticas (CAPs) de los pacientes sobre la hipertensión arterial se utilizó el test de Batalla: mediante preguntas sencillas, se analizó el grado de conocimiento de su enfermedad, asumiendo que un mayor conocimiento representa un mayor grado de cumplimiento. Es uno de los cuestionarios más habituales, por su alta sensibilidad, que se fundamenta en el conocimiento de la enfermedad, aunque tiene una baja especificidad (Zancheti, 2001). Según el consenso latinoamericano sobre hipertensión arterial, en el Ecuador tienen conocimientos sobre su enfermedad el $41 \%$ de pacientes (Zancheti, 2001). En Cuba el porcentaje de pacientes que conocen sobre su enfermedad alcanza el 56,8\% (Quintana y col., 2009). En el estudio realizado en Cuenca, el $51 \%$ de pacientes tenía conocimientos sobre su enfermedad antes de la intervención; después de ésta, el porcentaje se incrementó al 84\%, en tanto que el grupo control disminuyó del 50,23\% al 36,30\% (Tabla 6). Diversos factores pueden influir en el nivel de conocimientos, actitudes y prácticas (CAPs) con respecto a la hipertensión. Se ha demostrado que el nivel de escolaridad es importante puesto que las personas con instrucción superior alcanzan un porcentaje de CAPs muy superior al resto de grupos, lo que es lógico por la mayor posibilidad de comprender las orientaciones médicas. Las personas con vínculo laboral tienen una elevada adherencia comparada con los que no trabajan. Una baja red social es causa de no adherencia: la convivencia con otras personas se relaciona con una mejor adherencia, en tanto que el vivir solo ocasiona una pobre adherencia (López, 2007).

Tabla 6. Efectividad de la intervención en la mejora de las variables estudiadas en pacientes hipertensos de dos Centros del Área de Salud No2 Miraflores, Cuenca, 2011.

\begin{tabular}{|c|c|c|c|c|c|c|c|}
\hline \multirow[b]{2}{*}{ Variable } & \multicolumn{3}{|c|}{ Grupo Intervención } & \multicolumn{3}{|c|}{ Grupo Control } & \multirow[b]{2}{*}{$\mathrm{p}$} \\
\hline & Basal & $\begin{array}{l}\text { Post } \\
\text { interv. }\end{array}$ & $\begin{array}{c}\text { Post } \\
\text { interv. } \\
\text { - Basal }\end{array}$ & Basal & $\begin{array}{l}\text { Post } \\
\text { interv. }\end{array}$ & $\begin{array}{c}\text { Post } \\
\text { interv. } \\
\text { - Basal }\end{array}$ & \\
\hline PAS, media mmHg & 137,29 & 124,03 & $-13,26$ & 134,76 & 137,71 & 2,96 & 0,000 \\
\hline PAD, media mmHg & 80,48 & 78,29 & $-2,19$ & 78,31 & 81,57 & 3,26 & 0,023 \\
\hline Obesidad (IMC > 30), n \% & 30,00 & 39,00 & $-5,00$ & 27,85 & 27,37 & $-0,49$ & 0,000 \\
\hline Tabaco, n \% & 1,00 & 0,00 & $-1,00$ & 8,68 & 3,68 & $-4,99$ & 0,025 \\
\hline $\begin{array}{l}\text { Consumo de sal } \\
>6 \mathrm{~g} \mathrm{~d}^{-1}, \mathrm{n} \%\end{array}$ & 36,00 & 26,00 & $-10,00$ & 29,22 & 46,84 & 17,62 & 0,026 \\
\hline $\begin{array}{l}\text { Ingesta de alcohol } \\
>60 \mathrm{ml} \mathrm{d}^{-1}, \mathrm{n} \%\end{array}$ & 8,00 & 5,00 & $-3,00$ & 12,79 & 2,63 & $-10,15$ & 0,000 \\
\hline Sedentarismo, n \% & 52,00 & 33,00 & $-19,00$ & 47,03 & 44,21 & $-2,82$ & 0,000 \\
\hline $\begin{array}{l}\text { Adherencia al tratamiento, } \\
\text { n \% }\end{array}$ & 45,00 & 80,00 & 35,00 & 51,10 & 60,00 & 8,90 & 0,001 \\
\hline CAPs, (Si) n \% & 51,00 & 84,00 & 33,00 & 50,23 & 36,32 & $-13,91$ & 0,059 \\
\hline
\end{tabular}

\section{CONCLUSIONES}

Se identificaron los principales factores de riesgo asociados a los pacientes hipertensos, encontrándose significancia estadística $(\mathrm{p}<0,05)$ en la edad, el grado de instrucción escolar y la adherencia al tratamiento. Mediante la estrategia de Consultorios Clínicos Grupales, la intervención en los pacientes 
hipertensos fue efectiva para el descenso de las cifras de tensión arterial sistólica y diastólica, en la disminución de los factores de riesgo (excepto la obesidad) y en el aumento de la proporción de pacientes que cumplieron la adherencia al tratamiento, así como sus conocimientos, actitudes y prácticas (CAPs) relacionadas con la hipertensión arterial. De acuerdo a los resultados de este estudio, creemos que se puede implementar un modelo similar de APS para el manejo de la hipertensión arterial en otros Centros de Salud, con el fin de mejorar la adherencia al tratamiento y aumentar el grado de conocimiento de la enfermedad. Es fundamental la participación conjunta del equipo de salud y las organizaciones sociales, en este caso el "Club de hipertensos", con el fin de proteger, mejorar y recuperar la salud de las personas, las familias y las comunidades, asegurando a la población una atención de salud de calidad encaminada a lograr el Buen Vivir.

\section{AGRADECIMIENTOS}

Nuestro agradecimiento a la Dirección de Investigación de la Universidad de Cuenca (DIUC) que brindó el apoyo financiero y facilitó la ejecución de este proyecto. De igual manera, reiteramos nuestra gratitud a los señores ayudantes de investigación Christopher Cedillo, Laura Pesantez y Edison Calle por su incansable apoyo. Nuestra gratitud a la Dirección Provincial de Salud, a los médicos, personal de enfermería y directiva del Club de Hipertensos de los dos Centros de Salud, en especial al Dr. Juan Torres por su invalorable colaboración en la ejecución de este trabajo. Finalmente, dejamos constancia de nuestro agradecimiento a la Ingeniera Marlene Gia por su eficiencia en la parte administrativa.

\section{BIBLIOGRAFÍA}

Baena, J., J. Del Val, J. Pellegrina, 2005. Epidemiología de las enfermedades cardiovasculares y factores de riesgo en atención primaria. Rev. Esp. Cardiol., 58(4), 367-373.

Bertomeu, C., 2003. Guías sobre el tratamiento de la hipertensión arterial 2003: ¿aclaran o confunden? Rev. Esp. Cardiol., 56(10), 940-3.

Breilh, J., 2010. La epidemiología crítica: una nueva forma de mirar la salud en el espacio urbano. Salud Colectiva, 6(1), 83-101.

Coca Payeras, 2005. Evolución del control de la hipertensión arterial en Atención Primaria en España. Resultados del estudio Controlpres 2003. Hipertensión, 22(4), 172.

Colegio Americano de Cardiología, 2011. Consenso de Expertos sobre la hipertensión en ancianos. J. Am. Coll. Cardiol., 57, 2037-2114.

De Frutos, E., G. Lorenz, 2008. Cumplimiento terapéutico en pacientes con enfermedad cardiovascular. Clin. Invest. Arterioscl., 20(1), 8-13.

Díaz, M., 2002. Hypertension and obesity. J. Human Hyperten., 16(1), S18-S22.

Dosse, C., C. Bernardi, 2009. Factores asociados a la no adhesión de los pacientes al tratamiento de hipertensión arterial. Rev. Latino-Am. Enfermagem, 17(2), 1-7.

Ebrahim, S., F. Taylor, K. Ward, A. Beswick, M. Burke, G.D. Smith, 2011. Intervenciones sobre múltiples factores de riesgo para la prevención primaria de la cardiopatía coronaria. Cochrane Database of Systematic Reviews, Issue 1. Art. No.: CD001561. DOI: 10.1002/14651858.CD001561.

Fasce, E., 2000. Hipertensión arterial y enfermedad hipertensiva. Aspectos epidemiológicos. Enfermedades del corazón y de los vasos, 3a Ed., Editorial Mediterráneo, Santiago, Chile, 542546. 
Feinleib, H.H., P. McNamara, W. Castelli, 1983. Obesity as an independent risk factor for cardiovascular disease: a 26 year follow-up participants in the Framingham Heart Study. Circulation, 67,968-77.

Ferrera, N., D. Moine, D. Yanez, 2010. Hipertensión arterial: implementación de un programa de intervención de control y adherencia al tratamiento en un Centro de Atención Primaria de la Salud de la ciudad de Paraná. Rev. Fed. Arg. Cardiol., 39(3), 194-203.

Gómez-Marcos, M.A., L. García-Ortiz, L.J. González-Elena, E. Ramos-Delgado, A.M. GonzálezGarcía, J. Parra-Sánchez, 2006. Efectividad de una intervención de mejora de calidad en el control de la presión arterial en Atención Primaria. Rev. Clin. Esp., 206(9), 428-34.

Joint National Committee on Prevention, Detection, Evaluation and Treatment of High Blood Pressure, 2004. Guidelines. JNC 7 Report. En: http://www.nhlbi.nih.gov, Acceso octubre 2010.

INEC, 2011. Principales causas de mortalidad general en el año 2010. Anuario de estadísticas vitales. En: htpp//www.inec.gov.ec, Acceso septiembre 2011.

Consenso Latinoamericano sobre HTA, 2001. J. of Hypertens., 6(2), 1-22.

Jiang, H., L.G. Ogden, L.A. Bazzano, 2002. Dietary sodium intake and incidence of congestive heart failure in overweight US men and women. First National Health and Nutrition Examination Survey Epidemiologic Follow-up Study. Arch. Intern. Med., 162, 1619-1624.

Kaplan, N.M., 1994. Primary hypertension: Natural history, special populations and evaluation. Clinical hypertension, 6th Ed., Williams and Wilkins, Baltimore, 109-43.

Lamar, V., M. Hill, 2002; Estrategias eficaces de control de la presión arterial. Clínicas Cardiológicas de Norteamérica, 2, 327-336.

Limansky, R., J. Farías, M. Cámera, 2005. Multicenter study of arterial hypertension prevalence. 15th Scientific Meeting of the ISH, Melbourne, Resumen 2344.

López, F.V., 2002. Prevalencia de la hipertensión sistólica aislada en una población mayor de 60 años. Rev. Hipertens., 19(9), 384-389.

López, J., 2007. Programa para detección, evaluación y tratamiento del paciente hipertenso, 2a Ed., Litografía Proimpre C.A., Venezuela.

Loucks, E., 2011. Associations of education with 30 year life course blood pressure trajectories: Framingham Offspring Study. BMC Public Health, 11, 139.

Martorelly, R., A. Stein, 2003. El surgimiento de las enfermedades crónicas relacionadas con la dieta en los países en desarrollo. Conocimientos Actuales sobre Nutrición, GT: 723724, Publicación Científica y Técnica No. 592, 723-745.

Marso, S., B. Griffin, E. Topol, 2002. Cardiología. The Cleveland Clinic Foundation, Madrid, España, Marbán, S.L., 421-446.

Mazza, J., 2003. Mediciones antropométricas. Estandarización de las técnicas de medición, actualizada según parámetros internacionales. PubliCE Standard. En: http://www.sobreentrenamiento.com/publice/Articulo.asp?ida=197, Acceso: octubre 2010

Molina, J., 2008. Riesgo cardiovascular, ocupación y riesgos laborales en una población laboral de Catalunya. Med. Segur. Trab., LIV(212), 91-98.

Pérez García, M., R. Fernández Álvarez, P. Gayoso Diz, A. Martínez Vidal, 2002. Valoración del tratamiento farmacológico en un centro de salud en función de las patologías crónicas asociadas y grado de control de la HTA. Rev. Aten. Primaria, 29, 540-546.

Pimenta, E., C. Amodeo, S. Oparil, 2006. Hypertension in women. Int. J. Atheroscler., 3, 138-145.

Poveda, M., 2006. Características de la HTA en 50 pacientes del Centro de Salud No 3 del MSP Cuenca. Rev. Ateneo, 12(2), 22-26.

Quintana, C., J. Fernández, B. Rodríguez, 2009. Adherencia terapéutica farmacológica antihipertensiva en adultos de atención primaria y factores relacionados con su incumplimiento. Rev. Cubana Invest. Bioméd., 28(2), 4 págs. 
Rodríguez, C., 2009. Eficacia de una intervención educativa grupal sobre cambios en los estilos de vida en hipertensos en atención primaria: un ensayo clínico aleatorio. Rev. Esp. Salud Pública, 83(3), 441-452.

Rodríguez Chamorro, M., E. García, P. Amariles, 2008. Revisión de tests de medición del cumplimiento terapéutico utilizados en la práctica clínica. Rev. Aten. Primaria, 40(8), 413-417.

Rosas, M., G. Pastelín, G. Vargas-Alarcón, J. Martínez-Reding, C. Lomelí, C. Mendoza-González, J.A. Lorenzo, A. Méndez, M. Franco, L.G., Sánchez-Lozada, J. Verdejo, N. Sánchez, R. Ruiz, S.M. Férez-Santander, F. Attie, 2008. Guías clínicas para la detección, prevención, diagnóstico y tratamiento de hipertensión arterial sistémica en México. Archivos de Cardiología de México, 78(2), 5-57.

Saieh, C., C. Zehnder, 2008. Hipertensión arterial. Editorial Mediterráneo, Santiago, Chile, 37, 233235.

Sánchez, O., 2005. Estrategias para mejorar la adherencia terapéutica en patologías crónicas. Información Terapéutica del Sistema Nacional de Salud, 29(2), 40-48.

Valdéz, G., 2009. Sal e hipertensión arterial. Rev. Chil. Cardiol., 28, 107-114.

Valdivieso, M., 2008. Adherencia al tratamiento de la Medicina de Familia en pacientes con HTA, residentes en la parroquia Santa Ana de Cuenca, en el año 2008. Rev. Univ. Azuay, 50(8), 49-67.

Varo, J., A. Martínez, M. Martínez, 2003. Beneficios de la actividad física y riesgos del sedentarismo. Med. Clin., 121(17), 665-672.

Zancheti, A., 2001. Consenso Latinoamericano sobre Hipertensión Arterial. J. Hypertens., 6(2), 1-27. 